\title{
Metal coordination as a template strategy to make resilient woven materials
}

\author{
${ }^{a}$ Department of Chemistry, University of California-Berkeley, Materials Sciences Division, \\ Lawrence Berkeley National Laboratory, Kavli Energy NanoSciences Institute, Berkeley, California \\ 94720, United States \\ ${ }^{b}$ King Abdulaziz City for Science and Technology, Riyadh 11442, Saudi Arabia \\ Yuzhong Liu a and Omar M. Yaghi*,a,b \\ Received February 17, 2018 ; E-mail: yaghi@berkeley.edu
}

\begin{abstract}
Transition metal ions as a template method has been widely used in the field of supramolecular chemistry. The metal complexation is advantageous in making complex supramocluar architectures because it pre-organizes the ligands into a desirable orientation which faciliatates the following ring-closing reaction, with shorter synthetic steps and generally higher yield. In a similar fashion, this synthetic strategy has recently been adopted to make extended materials by linking the metal-coordinated building blocks with design principles of reticular synthesis. Individual building units are stitched together through strong covalent bond formation to yield long covalent molecular threads that are woven two- or three-dimensionally (2D or 3D), at regular intervals templated by the metal ions. For example, by linking functionalized tetrahedrally-shaped metal complexes with linear links through reversible imine bond formation, crystalline 3D covalent organic frameworks with diamond topology, COF-505 and COF-112, have been constructed by design. In particular, the metal templates can be post-synthetically removed so that the threads have high degrees of freedom to move in respect to each other, which leads to unusal mechanical properties of the woven materials.
\end{abstract}

\section{Introduction}

Metal coordination has been used extensively as a template strategy in the field of supramolecular chemistry. Preorganization of molecular components into a desirable orientation by metal complexation has allowed for the designed synthesis of interlocking molecules in high yields. Synthetic chemists have since learned to intertwine discrete molecular entities into entangled architectures with increasing complexity limited only by imagination. This template strategy has recently been implemented in the making woven extended structures. By linking building units that contain a metal complex core, two- and three-dimensional (2D and 3D) frameworks can be constructed where long threads of covalently linked molecules that are interwoven at regular intervals and templated by the metal ions. Upon demetalation, the threads have high degrees of freedom for spatial deviation

Corresponding Author: Omar M. Yaghi

Address: 602 Latimer Hall, Berkeley, CA 94720

Tel: 510-643-5507

Keywords: Transition metal-templated synthesis, knots and catenanes, extended frameworks, covalent organic frameworks, COFs, woven materials, weaving to take place between them while preserving the overall structure, endowing the material with exceptional mechanical properties and dynamics.

\section{Metal-templated synthesis of discrete interlocking molecules}

The designed synthesis of interlocking molecules (e.g. knots, catenanes and rotaxanes) is well documented in the chemical literature. ${ }^{1-9}$ The first proof-of-concept work in the field was reported in 1960 with the successful synthesis of a [2]catenane by statistical interlocking of molecular macrocycles. ${ }^{10}$ In 1964, the first directed synthesis of a catenane was demonstrated by introducing a covalent template to bring the two molecular components in place. ${ }^{11}$ Upon subsequent cleavage of this covalent template, the catenated molecule was obtained in higher yields as compared to the previously reported statistical approach. However, both the early statistical and directed synthetic approaches to mechanically interlocked molecules suffered from low overall yields and/or lengthy synthetic procedures. Catenanes thus remained in the realm of laboratory curiosities rather than 
molecular constructs that could be practically exploited.

The breakthrough in the field took place in 1983 when a metal-template approach was disclosed to construct higherorder supramolecular architectures. ${ }^{12}$ This enabled catenanes to be made on a significant scale for the first time. In the synthesis, the metal complex orients the individual ligands to ensure spatial overlap in order to create what we call crossing points (points of registry), which allows for efficient ring closure and the synthesis of such entangled architectures in high yield. Here, the approach employed a tetrahedrallycoordinated $\mathrm{Cu}(\mathrm{I})$ complex with two hydroxy-functionalized 2,9-diphenyl-1,10-phenanthroline ligands. The $\mathrm{Cu}(\mathrm{I})$ ion serves as the point of registry, and the ligands are positioned in an embracing fashion to facilitate efficient formation of the entanglement. Subsequent ring closing Williamson ether macrocyclization generated the [2]catenand in $42 \%$ yield. The $\mathrm{Cu}(\mathrm{I})$ ions could be removed quantitatively from the catenand with potassium cyanide to afford the metal-free [2]catenane (Fig. 1).
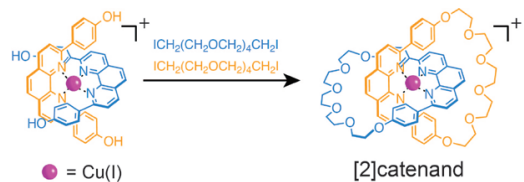

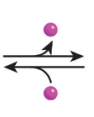

[2]catenand

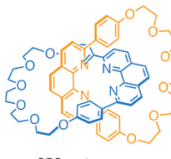

[2]catenane
Fig. $1 \mathrm{Cu}(\mathrm{I})$-bisphenanthroline-templated synthesis of a [2] catenane.

Developments in dynamic covalent bond forming reactions were also exploited towards the synthesis of interlocked molecules. Molecular Borromean rings were stitched together through the one-pot reaction of six dialdehydes, six diamines, and six $\mathrm{Zn}(\mathrm{II})$ ions using reversible imine formation under thermodynamic control (Fig. 2). ${ }^{3}$ The Borromean ring topology was confirmed by X-ray crystallography, which further corroborated that the combination of $\pi-\pi$ stacking interactions and metal coordination likely directed the assembly process. The $\mathrm{Zn}$ (II) templates could be removed from the structure by reduction of the imine bonds with sodium borohydride $\left(\mathrm{NaBH}_{4}\right)$, followed by treatment with

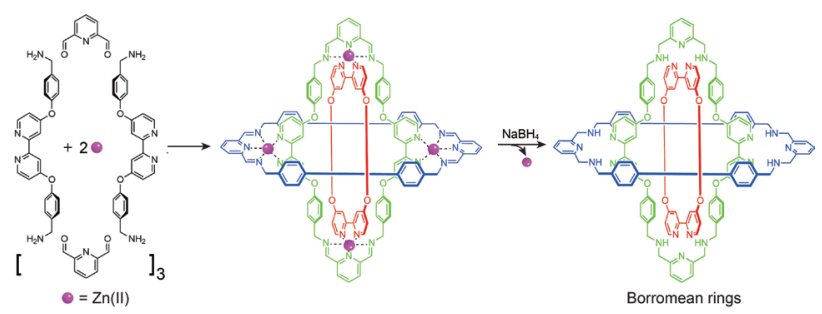

Fig. 2 Synthetic route for the formation of molecular Borromean rings. Atoms in red and blue rings are omitted for clarity; only the green ring contains all the atoms and bonds present in the macrocycles. ethylenediaminetetraacetic acid (EDTA) to afford the demetalated Borromean rings. ${ }^{13}$

In addition to higher yields and shorter synthetic routes associated with metal templated synthesis, specific orientation of the ligands due to complexation enables the rational design of synthetic pathways to more complex topologies and the ability of synthetic chemists to construct increasingly complex molecular structures has since then progressed remarkably resulting in a plethora of highly sophisticated supramolecular architectures. $^{2-9}$

It should be noted that these entangled molecular entities are held together mechanically, not chemically, which allows for large amplitude molecular motion of the individual units in respect to each other without breaking covalent bonds. On the molecular level, this approach has proved fruitful for the development of molecular machines ${ }^{14-15}$, muscles ${ }^{16}$ and rotors ${ }^{17-18}$ where during a switching process, only weak noncovalent bonds are broken and reformed again in a fully reversible and highly controllable fashion.

Towards this end, incorporation of interlocked molecules with specific properties (e.g. mechanical strength or elasticity) as building blocks to make extended structures with higherorder entanglement will lead to materials displaying interesting behavior originating both, from the intrinsic chemical nature of the molecular units themselves, as well as from emerging properties resulting from their global interlocking scaffold. ${ }^{19,20}$ As a consequence, potential applications of catenanes are no longer restricted to the realm of molecular chemistry, but can now further include extended structures such as covalent organic frameworks (COFs), which can display mechanical or dynamic properties of interest.

\section{Metal-templated synthesis of crystalline woven materials}

\subsection{Woven structures and their synthetic strategy}

Weaving of long threads is one of the oldest and most enduring methods of making fabrics that are so soft that they can be worn, yet robust enough to not easily be teared apart. Linking molecular building units by strong bonds through reticular synthesis into woven framework structures would represent a powerful strategy to transfer this concept into the realm of synthetic chemistry. This holds great promise to yield materials with exceptional mechanical properties and dynamics. Atomically defined woven materials have been long sought after but it remains a challenge to construct highly ordered weaving patterns and to assemble their regular crossings by design. 
Examples in chemical structures have been reported as woven or polycatenated networks. ${ }^{21-26}$ These examples while certainly interesting with respect to their unique structure types were not systematically targeted and are not the result of structural design but much rather products of fortuity. Furthermore, there is currently no strict definition in the literature as to what constitutes a building unit (thread) of woven or polycatenated structures. Here we will focus on the design and synthesis of networks where the interactions within each thread (chemical bonding) are sufficiently different from the interactions between them (weak, non-covalent interactions).

To successfully design the weaving of threads into $2 \mathrm{D}$ and 3D chemical structures, long covalently linked molecules (i.e., 1D units) must be able to cross at regular intervals. It would also be desirable if such crossings serve as points of registry, so that the threads can have many degrees of freedom to move away from and back to such points without collapsing the overall structure.

We envisioned that the aforementioned metal-templated synthetic strategy in conjunction with the design principles of reticular synthesis, which describes the linking of molecular building blocks to construct extended crystalline frameworks, could be employed to target such woven structures (Fig. 3).
Here again we start with a molecular metal complex with functionalized organic ligands, but instead of forming discrete molecules by non-propagating ring closing reactions, they are linked to form 2D and 3D framework structures through the formation of strong, directional bonds. The directionality of the bonds is crucial as, together with the adjustment of angles and metrics of the molecular constituents, it allows for the resulting woven structures to be designed with regard to their underlying topology.

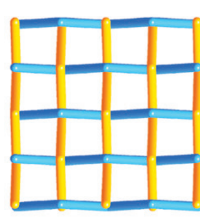

2D Weaving

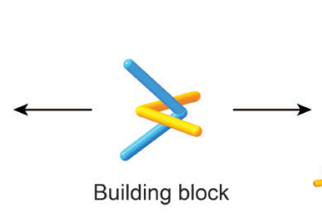

Fig. 3 Synthesis of 2D and 3D woven extended structures by linking molecular building blocks.

\subsection{Design and synthesis of crystalline woven covalent organic frameworks}

In order to construct a woven material, an aldehyde functionalized derivative of complex $\mathrm{Cu}(\mathrm{I})$-bis [4,4' -(1,10-phenanthroline-2,9-diyl)dibenzaldehyde ] tetrafluoroborate, $\mathrm{Cu}(\mathrm{PDB})_{2} \mathrm{BF}_{4}$, was chosen as the building
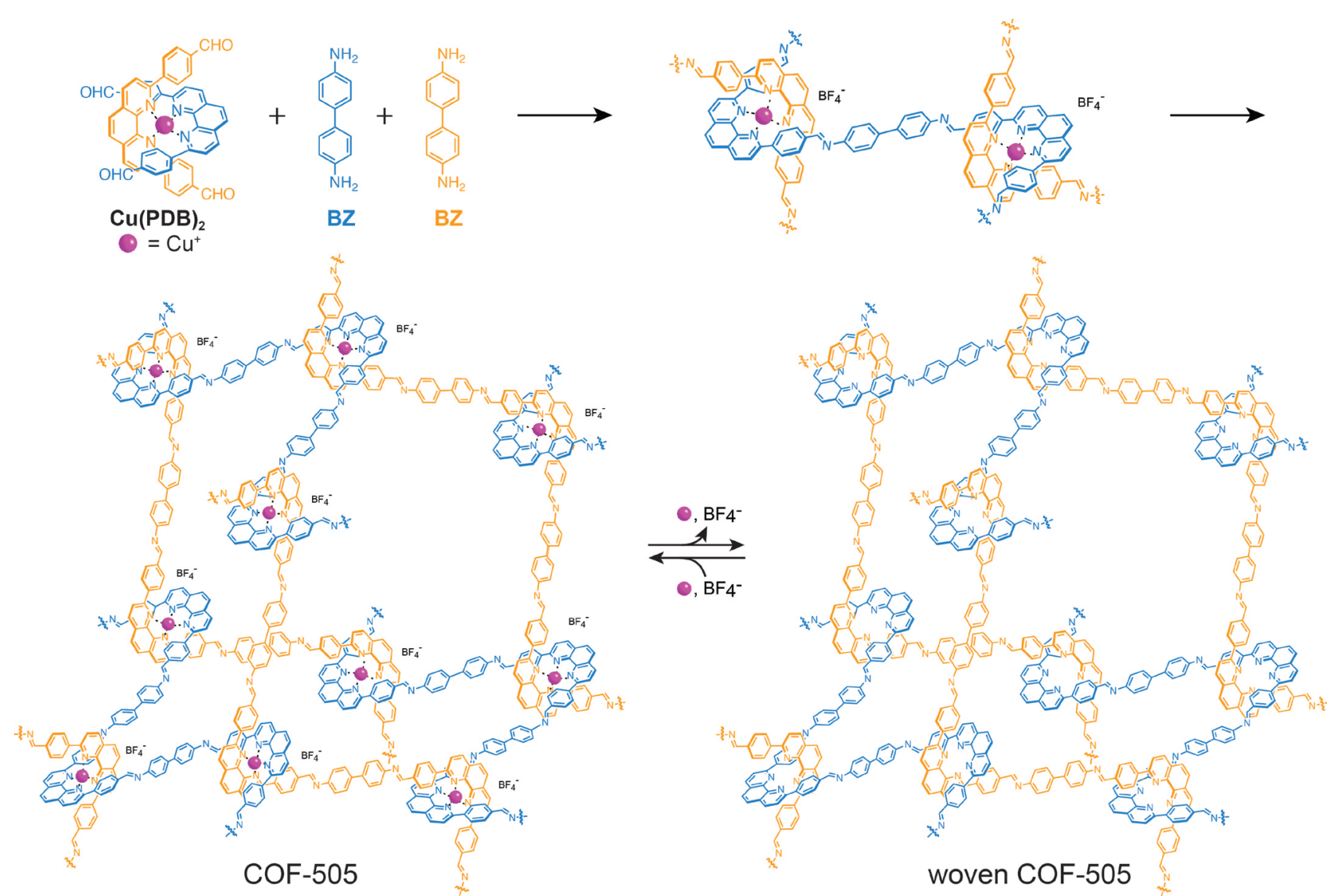

Fig. 4 By using $\mathrm{Cu}(\mathrm{I})$ as a template, COF-505, an extended crystalline woven structure, was constructed from linking building blocks by imine bond formation. The framework can be reversibly demetalated and remetalated. 
block. ${ }^{27}$ The $\mathrm{Cu}(\mathrm{I})$-bisphenanthroline core of the $\mathrm{Cu}(\mathrm{PDB})_{2}$ has been studied extensively as a discrete molecule for the formation of supramolecular complexes as mentioned before; however, previously the use of such complexes as templates to make extended structures had not been realized. The tolerance for robust reaction conditions makes this complex suitable for imine COF synthesis, especially in weak acidic conditions. The aldehyde groups in the para position of $\mathrm{Cu}(\mathrm{PDB})_{2}\left(\mathrm{BF}_{4}\right)$, were used to reticulate this building block with benzidine (BZ) to yield an imine-bonded framework composed of PDBBZ threads (Fig. 4). The orientation of the PDB units in a mutually interlacing fashion ensures that the threads produced from linking the building units are entirely independent, with the $\mathrm{Cu}(\mathrm{I})$ ions serving as templates (points of registry) to bring those threads together in a precise manner at well-defined intervals. The topology of this framework is dia, as expected from the principles of reticular chemistry.

COF-505, a three-dimensional extended framework, was constructed from helical organic threads with a woven crystal structure with atomic precision. The $\mathrm{Cu}(\mathrm{I})$ ions can be reversibly removed and added without loss of the overall COF structure. The threads in demetalated COF505 have many degrees of freedom for enormous deviations to take place without undoing the weaving of the overall structure, facilitated by the unique structure of weaving threads that easily 'zip' and 'unzip' at their points of registry. This dematalation is accompanied by a ten-fold increase in elasticity, and unlike the metalated crystalline form the demetalated material displays polymer-like elasticity. Upon remetalating, the crystallinity of the original as-synthesized structure can be fully restored.

In order to further expand the diversity of woven structures, a second woven framework, COF-112, was also synthesized by a similar metal coordination template strategy. ${ }^{28}$ Aminefunctionalized complexes with a cobalt bis(diiminopyridine) core, $\left[\mathrm{Co}\left(\mathrm{NH}_{2} \text {-DIP }\right)_{2}\right]$, were employed as the tetrahedral building blocks. The complex has a dihedral angle (between the two pyridine rings) of $80^{\circ}$, close to the angle of $90^{\circ}$ of an idealized dia topology in its highest symmetry embedding. In addition, tert-butyloxycarbonyl (Boc) groups were utilized to partially protect the primary amines in the starting materials, which increases the solubility of the starting reagents as well as intermediates, allowing for the maximum degree of error correction of the intermediates during the dynamic bond formation to yield extended frameworks.

The successful synthesis of COF-112 was carried out with 4-(tert-butoxycarbonylamino)-aniline (NBPDA), 2,6-pyridinedicarboxaldehyde (PCBA), and $\mathrm{Co}\left(\mathrm{BF}_{4}\right)_{2}$ in a one pot synthesis under solvothermal conditions. NBPDA and PCBA first undergo imine condensation to form the Bocprotected diiminopyridine ligand which quickly binds cobalt ions to form the Boc-protected precursor complex. The heatfacilitated in situ Boc-deprotection yields free amine groups to react with the remaining PCBA, forming the interlacing 1D zigzag polyimine chains in the extended COF-112 structure. The neighboring threads (blue and orange) are held woven at the coordinated cobalt ions as the point of registry.

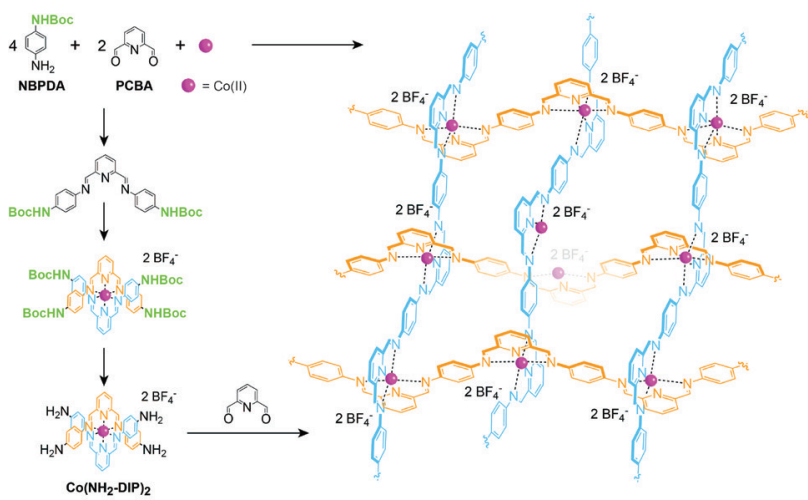

Fig. 5 One-pot synthetic route for the crystalline woven COF-112 by employing $\mathrm{Co}(\mathrm{II})$ ions as templates.

We note that in these COF structures, the woven long threads are comprised of only organic units linked through strong covalent bonds. This is important because after removing the metal ion templates, the bonding within each thread is remarkably stronger than the mechanical entanglements between them which ensures the chemical and structural integrity of the materials. The individual woven threads, therefore, can have high degrees of freedom for spatial deviations to move about the points of registry without collapsing the structures. Such freedom allows for reversible remetalation/demetalation, which exerts a reversible control over the mechanical properties of materials.

\section{Conclusion}

Transition metal ions as a templating strategy, widely utilized in supramolecular chemistry, has recently been adopted to make the first woven COFs. By reticulating the pre-organized ligands templated by metal complexes through strong covalent bonds, 3D crystalline frameworks (COF505 and COF-112) which consist of interlacing organic threads have been constructed. In these structures, metal ions serve as the points of registry where the threads cross over regularly; these templates can also be removed postsynthetically to obtain the woven materials, which allow for large degrees of freedom and structural motions between the 
individual threads to occur reversibly and repeatedly while maintaining the overall topology. This strategy of weaving organic threads and its successful implementation is the first step in the development of a large area of synthetic chemistry of materials with unique mechanical properties.

\section{Acknowledgements}

Y.L. acknowledges funding from the Philomathia Graduate Fellowship in the Environmental Sciences. Y.L. and O.M.Y. acknowledge BASF (Ludwigshafen, Germany), the Department of Energy (Office of Basic Science), and the King Abdulaziz City for Science and Technology for financial support.

\section{References}

1) C. Dietrich-Buchecker, J.-P. Sauvage, J. Am. Chem. Soc., 106, 3043 (1984).

2) C. Dietrich-Buchecker, J.-P. Sauvage, Angew. Chem. Int. Ed., 28, 189 (1989).

3) K. S. Chichak, S. J. Cantrill, A. R. Pease, S. H. Chiu, G. W. Cave, J. L. Atwood, J. F. Stoddart, Science, 304, 1308 (2004).

4) C. S. Wood, T. K. Ronson, A. M. Belenguer, J. J. Holstein, J. R. Nitschke, Nat. Chem., 7, 354 (2015).

5) N. Ponnuswamy, F. B. L. Cougnon, G. D. Pantoş, J. K. M. Sanders, J. Am. Chem. Soc., 136, 8243 (2014).

6) J. E. Beves, J. J. Danon, D. A. Leigh, J. F. Lemonnier, I. J. Vitorica-Yrezabal, Angew. Chem. Int. Ed., 54, 7555 (2015).

7) J.-F. Ayme, J. E. Beves, D. A. Leigh, R. T. McBurney, K. Rissanen, D. Schultz, Nat. Chem., 4, 15 (2011).

8) D. A. Leigh, R. G. Pritchard, A. J. Stephens, Nat. Chem., 6, 978 (2014).

9) J. J. Danon, A. Krüger, D. A. Leigh, J. F. Lemonnier, A. J. Stephens, I. J. Victorica-Yrezabal, S. L. Woltering, Science, 162, 159 (2017).

10) E. Wassermann, J. Am. Chem. Soc., 82, 4433 (1960).

11) G. Schill, A. Lüttringhaus, Angew. Chem. Int. Ed., 3, 546 (1964).

12) C. O. Dietrich-Buchecker, J. P. Sauvage, J. P. Kintzinger, Tetrahedron Lett., 24, 5095 (1983).

13) A. J. Peters, K. S. Chichak, S. J. Cantrill, J. F. Stoddart, Chem. Commun., 3394 (2005).

14) V. Balzani, M. Gómez-López, J. F. Stoddart, Acc. Chem. Res., 31, 405 (1998).

15) C. Tock, J. Frey, J. P. Sauvage, Transition Metal-Complexed Catenanes and Rotaxanes as Molecular Machine Prototypes in Molecular Switches, Second Edition, B. L. Feringa, W. R. Browne (ed), Wiley-VCH Verlag GmbH \& Co. KGaA, Weinheim, Germany, Chap. 4 (2011).

16) C. J. Bruns, J. F. Stoddart, Acc. Chem. Res., 47, 2186 (2014).

17) N. Koumura, R. W. J. Zijlstra, R. A. Van Delden, N. Harada, B. L. Feringa, Nature, 401, 152 (1999).

18) C. A. Schalley, K. Beizai, F. Vögtle, Acc. Chem. Res., 34, 465 (2001).

19) T. Takata, Polym. J., 38, 1 (2006).
20) Y. Okumura, K. Ito, Adv. Mater., 13, 485 (2001).

21) U. Lewandowska, W. Zajacczkowski, S. Corra, J. Tanabe, R. Borrmann, E. M. Benetti, S. Stappert, K. Watanabe, N. A. K. Ochs, R. Schaueblin, C. Li, E. Zashima, W. Pisula, K. Müllen, H. Wennemers, Nat. Chem., 9, 1068 (2017).

22) Y.-H. Li, C.-Y. Su, A. M. Goforth, K. D. Shimizu, K. D. Gray, M. D. Smith, H.-C. zur Loye, Chem. Commun., 14, 1630 (2003).

23) L. Han, Y. Zhou, Inorg. Chem. Commun., 11, 385 (2007).

24) A. M. Champsaur, C. Mézière, M. Allain, D. W. Paley, M. L. Steigerwald, C. Nuckolls, P. Batail, J. Am. Chem. Soc., 139, 11718 (2017).

25) Z. Wang, A. Blaszczyk, O. Fuhr, S. Heissler, C. Wöll, M. Mayor, Nat. Commun., 8, 14442 (2017).

26) F. L. Thorp-Greenwood, A. N. Kulak, M. J. Hardie, Nat. Chem., 7, 526 (2015).

27) Y. Liu, Y. Ma, Y. Zhao, X. Sun, F. Gándara, H. Furukawa, Z. Liu, H. Zhu, C. Zhu, K. Suenaga, P. Oleynikov, A. S. Alshammari, X. Zhang, O. Terasaki, O. M. Yaghi, Science, 351, 365 (2016).

28) Y. Zhao, L. Guo, F. Gándara, Y. Ma, Z. Liu, C. Zhu, H. Lyu, C. A. Trickett, E. A. Kapustin, O. Terasaki, O. M. Yaghi, J. Am. Chem. Soc. 139, 13166 (2017). 


\section{Profile}

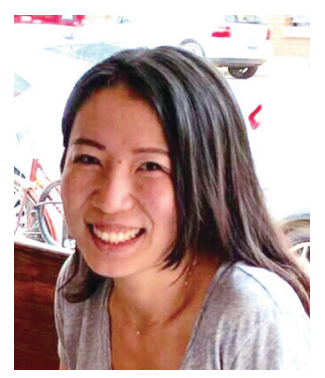

Yuzhong Liu grew up in Hangzhou, China and went on to pursue her B.Sc. degree in Chemistry at University of Michigan, Ann Arbor where she discovered snow and her passion for chemistry. After graduation, she took a gap year and developed chemical tools for neurodegenerative diseases such as Alzheimer's Disease under the guidance of Professor Mi Hee Lim. In 2013, Yuzhong started graduate school at UC Berkeley and joined Professor Omar Yaghi's group. Her research interest now focuses on the design and synthesis of crystalline woven covalent organic frameworks.

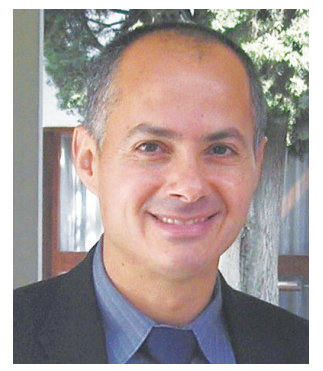

Omar M. Yaghi received his Ph.D. from the University of Illinois-Urbana with Prof. Walter G. Klemperer, and studied as an NSF Postdoctoral Fellow at Harvard University with Prof. Richard Holm. $\mathrm{He}$ is currently the James and Neeltje Tretter Chair Professor of Chemistry at UC Berkeley and a Senior Faculty Scientist at Lawrence Berkeley National Laboratory. He is also the Founding Director of the Berkeley Global Science Institute as well as the Co-Director of the Kavli Energy NanoScience Institute and the California Research Alliance by BASF. He is interested in the science of building chemical structures from organic and inorganic molecular building blocks- a field known as Reticular Chemistry, to make extended porous structures, such as MOFs, ZIFs, and COFs. 